\section{PWE-009 THE SHEFFIELD GASTROSTOMY SCORE (SGS): VALIDATION STUDY AT A LONDON DISTRICT GENERAL HOSPITAL}

Alasdair Macrae*, Claire Patton, William Hunter, Peter Foulser, Christopher Alexakis. Department of Gastroenterology, Kingston Hospital NHS Foundation Trust, London, UK

\subsection{6/gutjnl-2019-BSGAbstracts.340}

Introduction Insertion of percutaneous endoscopic gastrostomy (PEG) tubes allows long-term enteral feeding in selected patient groups, but the procedure is associated with not insignificant risks ${ }^{1}$. The Sheffield Gastrostomy Score (SGS) was developed to predict and quantify 30-day all cause mortality amongst patients undergoing PEG insertion based on the patient's age and serum albumin, but as yet has not been robustly validated in wider populations ${ }^{2}$.

Methods Having been identified on an endoscopy database, the clinical records of all patients who underwent PEG tube insertion at a London District General Hospital between August 2013 and August 2018 were retrospectively examined. Data collected for the study included basic demographics (including age and sex), biochemistry (including nearest albumin level prior to PEG procedure), and 30-day mortality as recoded on the hospital electronic record system. The SGS was calculated for each case with age scoring 0 or $1(<65 / \geq$ 65 years) and albumin scoring 0,1 or 2 ( $\geq 35,25-34$ and $<25 \mathrm{~g} / \mathrm{L})$. Observed 30-day mortality rates were then compared to those predicted by the SGS using the $\chi^{2}$ test.

Results 200 PEGs were inserted over this period of time with complete data available from 177 cases (89\%). 59\% of patients were male. Mean age of patients undergoing PEG insertion was 75 years. Mean pre-PEG albumin level was 27 g/L. Thirty-day all cause mortality for the whole cohort was 7.3\%. Observed and expected 30-day all cause mortality rates given SGS are demonstrated in Table 1.

$\begin{aligned} & \text { Abstract PWE-009 Table } 1 \\
& \text { mortality in patients undergoing PEG }\end{aligned}$
\begin{tabular}{lllllll} 
SGS & No. & No. & Observed & Expected & $\chi^{2}$ & p-value \\
& patients & deaths & & & & \\
\hline 0 & 5 & 0 & $0.0 \%$ & $0.0 \%$ & NA & NA \\
1 & 27 & 2 & $7.4 \%$ & $7.0 \%$ & 0 & 1 \\
2 & 97 & 5 & $5.2 \%$ & $21.3 \%$ & 15.4 & $<0.001$ \\
3 & 48 & 6 & $12.5 \%$ & $37.3 \%$ & 24.7 & $<0.001$ \\
\hline
\end{tabular}

Conclusion The SGS may over estimate 30-day mortality in patients undergoing PEG insertion, particularly those with scores of two or greater. Whereas these findings may reflect a difference in patient sample, further validation studies of the SGS are warranted.

\section{REFERENCES}

1. NCEPOD. National confidential enquiry into patient outcome and death: scoping out practice. London: National Confidential Enquiry into Patient Outcome and Death, 2004

2. . Kurien $M$, Robson $H E$, Leeds JS, et al External validation of a prognostic scoring system for percutaneous endoscopic gastrostomy (PEG) Gut 2011;60:A17.

\section{PWE-010 INTRODUCTION OF A PRIMARY CARE DIETETICS SERVICE FOR FUNCTIONAL GUT DISORDERS}

Sean Mole*, Natasha Rich, Nicholas Alexander Kennedy, James Goodhand, Tariq Ahmad, Chris Calvert. Department of Gastroenterology, Royal Devon and Exeter NHS Foundation Trust, Exeter, UK

\subsection{6/gutjnl-2019-BSGAbstracts.34}

Gastrointestinal symptoms constitute about $10 \%$ of all presenting complaints reported in new primary care appointments. We have recently introduced a calprotectin-based referral pathway for young adults presenting to primary care with GI symptoms: general practitioners can refer individuals with normal coeliac serology and negative stool calprotectin test straight to our dietician-led community clinic, which was previously only accessible following secondary care referral. We sought to define the caseload referred to- and the effectiveness of, our primary care dietician service.

Methods We conducted a prospective observational cohort service of patients referred to our new clinic between April 2018 and Jan 2019. Patients with coeliac disease and possible inflammatory bowel disease were excluded using serological tests and stool calprotectin, respectively. Symptoms were assessed using a symptom scale (SS) modified from the Gastrointestinal Symptom Rating Scale for irritable bowel syndrome. Patients also completed the Patient Health Questionnaire-12 Somatic Symptom (PHQ-12) scale. Personalised dietary treatments were instituted and patients were followed up after 8 weeks. Response was assessed using the symptom score and the dietitian assessment. Treatment failure was defined by a static symptom score and dieticians global assessment. Comparisons were made between referrals made to the service from primary compared with secondary care.

Results During the 9-month inclusion period our dietician treated 152 patients: 43\% (66/152) were referred from primary care, and 78\% (119/152) female. Referred primary care patients were younger (median (IQR) 30 (2-9) years vs $46(3-3)$ years, $\mathrm{p}<0.001)$ than patients referred from secondary care. Overall, the presenting symptoms were similar between groups. A low FODMAP diet was advised in $82 \%$ $(122 / 148)$ people, with other dietary advice given to $18 \%$ $(26 / 148)$. In 4 people dietary therapy was refused or felt to be inappropriate. Follow-up data was available in 94 patients: $65 \%(61 / 94)$ were deemed to have had a successful intervention. Rates of success were similar for primary $(67 \%)$ and secondary care referrals $(63 \%), p=0.83$. None of age, sex, somatisation score and screening tests for anxiety and depression were associated with differences in proportions with successful intervention.

Conclusions Overall, dietary therapies were effective in the management of about two-thirds of patients referred with gastrointestinal disorders. Patients referred direct from primary care were typically younger than those referred from secondary care. We plan to develop the service further by signposting referred patients with high PHQ-12 scores to our integrated depression and anxiety and psychology services. 